\title{
Leidenfrost Pattern Formation and Boiling
}

\author{
Prasanth Prabhakaran ${ }^{1,2,3} \cdot$ Alexei Krekhov ${ }^{1}$. Eberhard Bodenschatz ${ }^{1,3,4}$. \\ Stephan Weiss ${ }^{1,5}$ (DD
}

Received: 31 December 2018 / Accepted: 30 March 2019 / Published online: 9 April 2019

(c) The Author(s) 2019

\begin{abstract}
We report on Leidenfrost patterns and boiling with compressed sulfur hexafluoride $\left(\mathrm{SF}_{6}\right)$. The experiments were carried out in a large aspect ratio Rayleigh-Bénard convection cell, where the distance between the horizontal plates is comparable with the capillary length of the working fluid. Pressures and temperatures were chosen such that the bottom plate was above and the top plate was below the liquid-vapor transition temperature of $\mathrm{SF}_{6}$. As a result, $\mathrm{SF}_{6}$ vapor condenses at the top plate and forms drops that grow in size. Leidenfrost patterns are formed as the drops do not fall but levitate by the vapor released in the gap between the hot bottom plate and the colder drops. When the size of these drops became too large, one or more vapor bubbles - chimneys - form inside them. We determine the critical size for the formation of a chimney as a function of the capillary length. For even larger drops and extended puddles many disconnected chimneys occur that can grow to sizes large enough for the formation of new drops inside them. By varying the temperatures and the pressure in the system, we observe various such patterns. When the area covered by a puddle becomes large it touches the hot bottom plate locally and boils off rapidly. This can be attributed to a local reduction of the bottom plate surface temperature below the Leidenfrost temperature.
\end{abstract}

Keywords Leidenfrost patterns - Pattern formation - Phase transition - Surface tension · Rayleigh-Taylor instability $\cdot$ Fluids

Electronic supplementary material The online version of this article (https://doi.org/10.1007/s10955-01902283-7) contains supplementary material, which is available to authorized users.

Stephan Weiss

stephan.weiss@ds.mpg.de

1 Max Planck Institute for Dynamics and Self-Organization, 37077 Göttingen, Germany

2 Department of Physics, Michigan Technological University, Houghton, MI 49931, USA

3 Institute for the Dynamics of Complex Systems, University of Göttingen, 37073 Göttingen, Germany

4 Laboratory of Atomic and Solid-State Physics and Sibley School of Mechanical and Aerospace Engineering, Cornell University, Ithaca, NY 14853, USA

5 Max Planck - University of Twente Center for Complex Fluid Dynamics, Göttingen, Germany 


\section{Introduction}

We dedicate this paper to Pierre Hohenberg, a great scientist, scholar and friend. We were always impressed by his openness to new ideas, creativity and scientific rigor. The topic of this paper combines critical phenomena and pattern formation, two fields that were at the center of Pierre's scientific life.

A liquid drop placed on a surface, sufficiently hotter than its boiling temperature, may levitate when the pressure from evaporation of the drop overcomes its weight. This phenomena was described in detail by Johann Gotlob Leidenfrost in 1756 [1], and is called the Leidenfrost effect. The vapor layer underneath the drop creates a thermally insulating cushion that limits the heat transport and prevents the nucleation of bubbles inside the drop. As a result, the drop will not boil, but rather slowly shrink due to evaporation. The very same mechanism also occurs in more extended liquid layers or with hot objects inside a liquid, and is often also referred as film boiling [2,3]. The Leidenfrost effect plays an important role in heat transfer applications involving boiling, as the presence of a vapor layer between a liquid and a hot substrate severely restricts the heat transfer from the substrate to the liquid. The sudden reduction of the heat transport due to the formation of an insulating vapor layer for larger liquid volumes, is termed boiling crisis [4]. For a detailed account on the dynamics associated with the Leidenfrost effect see, e.g., [2,5].

The Leidenfrost effect occurs when the substrate temperature is greater than a critical temperature called the Leidenfrost temperature $T_{L}$. This was demonstrated by measuring the lifetime of a drop as a function of the substrate temperature [5]. The Leidenfrost temperature depends on the pressure [6] as well as on the nature of the substrate [7]. The shape of a levitating Leidenfrost drop is governed by surface tension, pressure due to evaporation, and gravity. Surface tension is dominant in drops smaller than the capillary length of the liquid, $\lambda=\sqrt{\sigma / g\left(\rho_{l}-\rho_{v}\right)}$, where $\sigma$ denotes the surface tension of the liquid, $g$ the gravitational acceleration, and $\rho_{l}, \rho_{v}$ the density of the liquid and the surrounding vapor, respectively. Hence drops significantly smaller than the capillary length acquire a nearly spherical shape, while drops larger than the capillary length flatten out due to gravity, becoming pancakeshaped [2].

Various aspects of Leidenfrost drops have been studied in the past, such as the impact of cold drops on a hot surface [8,9], or their shape as a function of size (see [2] for review). The liquid-vapor interface at the bottom of a Leidenfrost drop exhibits interesting dynamics due to the pressure and the flow generated underneath. For drops larger than the capillary length, the thrust from the vapor layer beneath the drop cannot be ignored. This results in the formation of small vapor cavities underneath the drop [2]. Drops of different sizes on hot surfaces have been investigated for different fluids at atmospheric pressure [5]. These experiments show that above a critical size of a drop (we call it a puddle), the bottom liquid surface of the puddle becomes unstable and a chimney with gas is formed that rises at the center of the puddle and making a liquid dome at its top. Further growth continues until the dome bursts, and the remaining liquid puddle rearranges to fill in the void. The formation of these chimneys are interpreted as an inverse Rayleigh-Taylor instability as the less denser fluid is pushing against the denser fluid from underneath $[2,5,10]$. The instability occurs when the size of the puddle is larger than a certain critical size $R_{c} \approx 4 \lambda$ (for a circular puddle) with the thickness of the puddle $h \approx 2 \lambda[5,10,11]$. A further increase in the size of the drop results in the formation of multiple chimneys [2,5]. Not many investigations have focused on the dynamics of multiple chimneys in a single Leidenfrost drop. The present work fills partly this gap of knowledge. 
In this article, we report experimental results on Leidenfrost drops and puddles in a Rayleigh-Bénard setup, where a fluid is confined between a hot bottom and a cold top horizontal plate. We report the effect of the size of a liquid puddle on the dynamics of chimney formation. In particular, we report the results on the dynamics associated with multiple chimney formation and how these chimneys interact. The manuscript has four sections. In the next section, we discuss the experimental setup, followed by experimental observations and a brief summary.

\section{Experimental Setup}

A schematic of the experimental setup is shown in Fig. 1. The experiments were conducted in a high-pressure Rayleigh-Bénard convection setup that has previously been used to study pattern formation in single phase fluids [12-14]. A detailed description of the setup is given in [12]. The heart of the setup is the convection cell, which consists of a top plate made out of monocrystalline sapphire and a monocrystalline silicon bottom plate. Each of these circular plates is $9.5 \mathrm{~mm}$ thick and $101 \mathrm{~mm}$ in diameter. Their surfaces were flat to one-half of the wavelength of red light $(633 \mathrm{~nm})$ and polished to a mirror finish.

The bottom plate was heated to a temperature $T_{b}$ using an ohmic foil heater and the top plate was cooled to a temperature $T_{t}$ by circulating water on its surface. The two plates were parallel up to twice the wavelength of light $(633 \mathrm{~nm})$ over the entire visible diameter of the cell. The vertical distance between the two plates was $H=0.46 \pm 0.05 \mathrm{~mm}$. The cell had a circular planform of diameter $80 \mathrm{~mm}$ (aspect ratio $\approx 174$ ), with side walls made out of stacked paper slips. The top and bottom plate temperatures were regulated using proportional-differential feedback. Note that the stability of the bottom and top plate temperatures strongly depended on the dynamics of the fluid. When only a little amount of liquid was present the pattern were rather stable and fluctuations in the bottom and top plate temperature were not more than $\pm 10 \mathrm{mK}$. However, with more dynamics in the system, when more liquid was present, the fluctuations increased to $\pm 100 \mathrm{mK}$ and even reached $\pm 300 \mathrm{mK}$ when boiling occurred. The convection cell was attached to a pressure regulation vessel that kept the pressure inside the cell constant to within \pm 20 mbar.

We use sulfur hexafluoride $\left(\mathrm{SF}_{6}\right)$ as the working fluid. It has a critical pressure $P_{c}=$ $37.55 \mathrm{bar}$ and critical temperature $T_{c}=45.573^{\circ} \mathrm{C}$ [15]. Experiments were conducted at different pressures below $P_{c}$ and conditions where the top plate was colder and the bottom plate warmer than the corresponding saturation temperature $T_{s}$ (see Table 1). The cell is connected to an $\mathrm{SF}_{6}$ reservoir for pressure regulation, such that at a given pressure the amount of $\mathrm{SF}_{6}$ inside the cell is given by $T_{t}$ and $T_{b}$. Thus, the control parameters in our experiments are the pressure as well as $T_{t}$ and $T_{b}$. The pressure sets the fluid properties at the liquid-vapor interface, while the temperatures $T_{t}, T_{b}$ and the corresponding saturation temperature $T_{s}$ determined the amount of liquid in the system. When $T_{t}$ was closer to $T_{s}$, the cell was mostly filled with vapor and the amount of liquid is small. When $T_{b}$ was closer to $T_{s}$, the amount of liquid was large. In this respect it is helpful to consider the temperature fraction $\phi=\left(T_{b}-T_{s}\right) /\left(T_{b}-T_{t}\right)$. The cell is completely filled with vapor for $\phi \geq 1$ and completely filled with liquid for $\phi \leq 0$.

Images were taken from the top using a Phantom 65 Gold high-speed camera operated at a resolution of $2048 \times 2048$ pixels, with the focus set in order to image the middle of the cell. The maximum frame rate at this resolution was 140 frames per second. This enabled us 


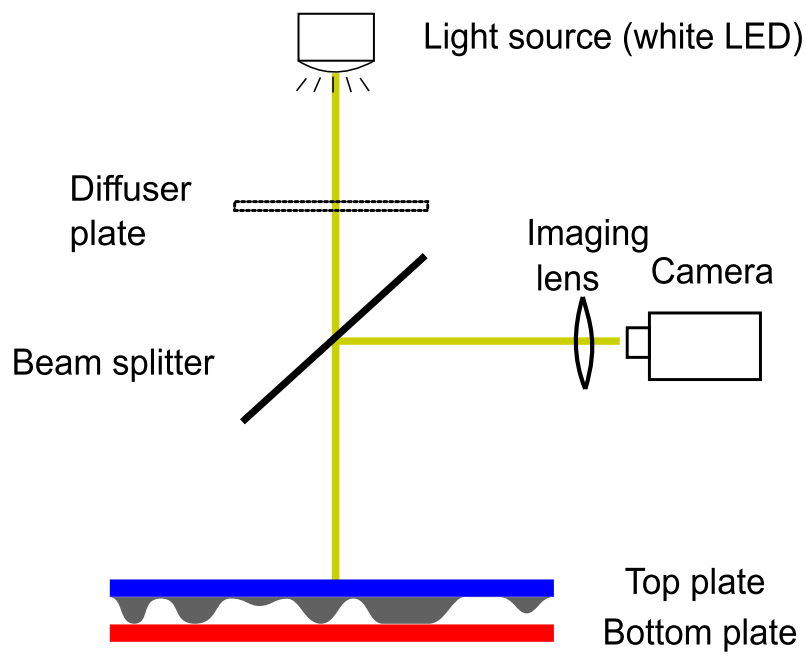

Fig. 1 A schematic of the convection cell and the visualization strategy

Table 1 Experimental conditions and material parameters

\begin{tabular}{llllllllll}
\hline $\begin{array}{l}P \\
\text { (bar) }\end{array}$ & $\begin{array}{l}T_{t} \\
\left({ }^{\circ} \mathrm{C}\right)\end{array}$ & $\begin{array}{l}T_{b} \\
\left({ }^{\circ} \mathrm{C}\right)\end{array}$ & $\begin{array}{l}T_{S} \\
\left({ }^{\circ} \mathrm{C}\right)\end{array}$ & $\phi$ & $\begin{array}{l}\sigma \cdot 10^{3} \\
(\mathrm{~N} / \mathrm{m})\end{array}$ & $\begin{array}{l}\rho_{l} \\
\left(\mathrm{~kg} / \mathrm{m}^{3}\right)\end{array}$ & $\begin{array}{l}\rho_{v} \\
\left(\mathrm{~kg} / \mathrm{m}^{3}\right)\end{array}$ & $\begin{array}{l}\lambda \\
(\mathrm{mm})\end{array}$ & $\begin{array}{l}T_{L} \\
\left({ }^{\circ} \mathrm{C}\right)\end{array}$ \\
\hline 23.72 & 24.0 & 28.0 & 25.16 & 0.71 & 1.614 & 1338.5 & 225.51 & 0.385 & 26.2 \\
24.00 & 24.0 & 28.0 & 25.65 & 0.59 & 1.564 & 1332.9 & 229.21 & 0.380 & 26.7 \\
28.40 & 32.0 & 40.0 & 32.97 & 0.88 & 0.8662 & 1238.6 & 295.19 & 0.306 & 33.7 \\
31.17 & 36.0 & 40.0 & 37.12 & 0.72 & 0.5146 & 1169.6 & 348.16 & 0.253 & 37.6 \\
31.45 & 36.0 & 40.0 & 37.52 & 0.62 & 0.4831 & 1162.0 & 354.19 & 0.247 & 38.0 \\
31.72 & 36.0 & 40.0 & 37.91 & 0.52 & 0.4523 & 1154.3 & 360.41 & 0.241 & 38.4 \\
34.21 & 40.0 & 42.0 & 41.33 & 0.34 & 0.2060 & 1073.9 & 427.53 & 0.180 & 41.6
\end{tabular}

Pressure in the cell $P$, top and bottom plate temperatures $T_{t}$ and $T_{b}$, respectively. Saturation temperature $T_{S}$ for a given pressure $P$ and corresponding temperature fraction $\phi$, surface tension $\sigma$ and densities of the liquid $\rho_{l}$ and vapor $\rho_{v}$ phases, taken from [15]. Leidenfrost temperature $T_{L}$ estimated from the relation obtained from the measurements for four organic liquids in [6]

to capture the fast dynamics that occurred during the merging and the boiling of liquid drops and puddles.

\section{Results}

In our experiment we have three independent control parameters that determine the state of the system. These are (i) the pressure $P$, (ii) the temperature fraction $\phi$, and (iii) the temperature difference $\Delta T$ between the bottom and top plates. Setting the pressure also sets the saturation temperature $T_{S}$ and thus the fluid properties like density difference between the liquid and the gas or the surface tension at the gas-liquid interface. The temperature fraction $\phi$ controls the ratio between the liquid and the gas phase in the cell. The last control parameter, the temperature difference, controls the strength of the thermal driving in the gas and the 
liquid as well as the evaporation and condensation rates. In this article we do not explore the whole 3-dimensional phase space but rather describe various phenomena and discuss their dependency on the control parameters. Nevertheless, in the following we present our observed phenomena and discuss in detail their dependency on the temperature fraction and the fluid properties.

\subsection{Development and Shape of Drops}

The bottom (top) plate was heated (cooled) to the desired temperature. As $T_{t}$ decreased below $T_{s}$, liquid $\mathrm{SF}_{6}$ condensed underneath the cold top plate, forming a thin liquid layer. This layer grew in thickness and underwent a Rayleigh-Taylor like instability $[16,17]$ in the form of periodic undulations. Regions of maximal layer thickness grew towards the bottom and appeared as drops in the camera image.

We did not have lateral optical access to the convection cell in our experiment. Therefore, we are uncertain whether the object that appeared as drops in the camera image were free drops, or whether they were still connected to the thin liquid layer underneath the top plate. According to experiments and theory for freely levitating Leidenfrost drops, the thickness of a small drop $(R \ll \lambda)$ is $h \approx 2 R$ and the thickness of a large drop $(R \gg \lambda)$ is $h \approx 2 \lambda$ $[2,5,10,11]$. On the other hand, for a drop to detach from the top plate its radius must exceed the capillary length $\lambda$. We obtain a better estimate by calculating the shape of a sessile drop not attached to the top plate and a hanging drop that is attached to the top. For this, we calculate the drop profile $z=z(r)$ in a vertical cross-section by balancing the forces normal to the drop surface (Young-Laplace relation)

$$
\sigma \kappa+\left(\rho_{l}-\rho_{v}\right) g z=P_{0}, \quad \kappa=\frac{1}{r} \frac{d}{d r}\left(r \frac{|d z / d r|}{\sqrt{1+(d z / d r)^{2}}}\right),
$$

where $\kappa$ is the local surface curvature calculated from the axial profile $z(r)$ (see, e.g., [11]), and $P_{0}$ is a reference pressure at the top of the drop $z=z_{\max }$. We have numerically integrated Eq. (1) for the cases of a drop sitting on the bottom plate not attached to the top and the case for a drop hanging from the top plate. Example profiles for both cases are shown in Fig. 2a. With this we can estimate the height of sessile drops of different sizes under experimental conditions. For almost all conditions studied in this paper, the thickness of large Leidenfrost drops was comparable to the height of the cell $H$. We thus conclude that the observed drops were connected to the liquid layer underneath the top plate.

Due to refraction, drops appear different at different stages of their development when imaged from the top. Examples are shown in Fig. 2c. The drop on the left (marked by a red arrow) and right (marked by a green arrow) are of the same visible size, but the left drop has a clear black rim around it, while for the drop on the right, the boundary of the drop is not well defined. We interpret this as the drop on the right having a much smaller vertical extent and thus does not reach the bottom plate yet, whereas the left drop extends down to the bottom plate where its shape is influenced by the evaporation pressure, i.e., the Leidenfrost effect. Figure $2 b$ shows the profiles of hanging drops of different sizes. We note that for the calculations using Eq. (1), any evaporation pressure or hydrodynamic interaction with the vapor was neglected. Note, that an apparent radius of the hanging drops - where the curvature of the profile $z(r)$ changes sign - increases slowly as the volume of the drop increases.

We estimate the Leidenfrost temperature $T_{L}$ for $\mathrm{SF}_{6}$ based on a dimensionless correlation found in the measurements for four organic liquids in [6]. There, the curves $T_{L}(P)$ for Freon C51-12, Freon 113, carbon tetrachloride and chloroform were obtained for pres- 


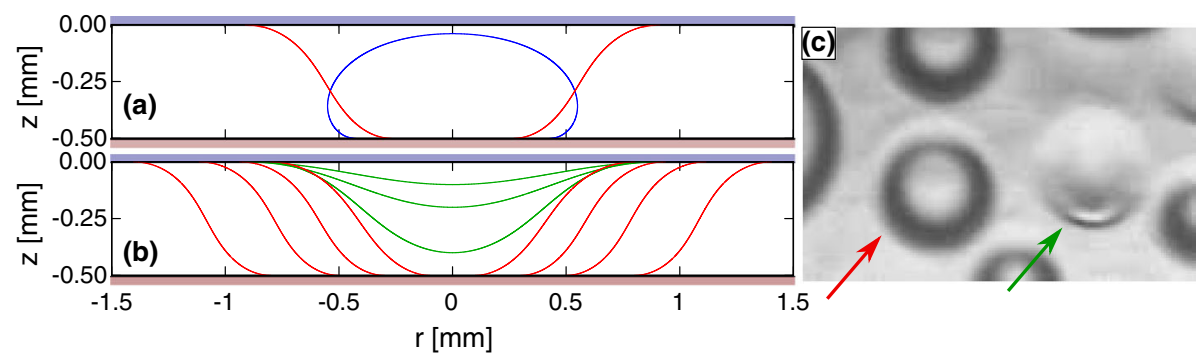

Fig. 2 a Mid-plane cross-sections for a sessile drop (blue) and a drop of similar size that is connected to the top plate (blue). Both profiles were calculated based on Eq. (1). b Cross-section profiles for drops of various extents hanging from the top plate. Cross-sections in green mark drops that do not extent to the bottom plate. Red cross sections mark drops that extend to the bottom plate. c Optical image showing two hanging drops of similar lateral size. The one on the right (green arrow) does not extend to the bottom plate (green curves in b). The left drop (red arrow) extends to the bottom plate (red curves in b). The z-range extends over the size of the experimental cell. Top and bottom plates of the cell are shown in $\mathbf{a}$ and $\mathbf{b}$ as light blueish and brownish thick lines (Color figure online)

sures up to the critical pressure $P_{c}$ and the relation $T_{s} / T_{L} \propto P / P_{c}$ has been found. For our experimental conditions we can estimate the Leidenfrost temperature using the relation $T_{S} / T_{L}=1-0.167\left(1-P / P_{C}\right)^{1.41}[6]$. According to these estimates the bottom plate temperatures in our experiments were above $T_{L}$ for all experimental condition reported here.

\subsection{Drops and Puddles at Different Temperature Fraction $\phi$}

Figure 3 shows Leidenfrost drops at various conditions. Most notably, the temperature fraction $\phi$ decreases, i.e., the amount of liquid in the cell increases from (a) to (d). Figure 3a shows typical drop patterns at large $\phi$, i.e., small amount of liquid. All drops are of similar size, are arranged rather regularly in space, and show very little dynamics. As the translational symmetry is lost over rather short distances no clear peaks are observed in the Fourier transform but rather a circle corresponding to a wavelength of $2.6 \mathrm{~mm}$. In order to reveal the local symmetry, we have calculated the Voronoi lattice for the drop pattern in Fig. 3a. Out of the 161 identified Voronoi cells, we counted 120 hexagons, 30 pentagons and 11 heptagons, suggesting a predominantly hexagonal arrangement that are disturbed by multiple pentahepta-defects [18]. At this point it is not well understood what causes the periodicity and stability of the patterns. It is likely that the thermally driven convection flow of the gas results in regular separation of drops. Furthermore, it has been shown [19] that a Rayleigh-Taylor unstable thin liquid film can be stabilized under the presence of a strong vertical temperature gradient. In this case, the evaporation (condensation) rate is enhanced when the liquid-vapor interface deforms and increases (decreases) its thickness locally due to perturbations. As a result, hexagonal stable undulations are expected and were found in simulations in [19]. We thus believe that the Leidenfrost effect plays only a minor role in dictating the organization of the drops in Fig. 3a. We refrain from a deeper analysis of the observed pattern and we refer the reader to an upcoming paper that is currently under preparation.

With increasing amount of liquid (decreasing $\phi$ ), these small drops grow in size, and as a consequence Leidenfrost effect starts influencing the dynamics associated with the drop and their sizes were distributed more inhomogeneously. We observed that the size of a single drop did not change in time, suggesting a balance between condensation and evaporation. However, a drop might jitter slightly due to the flow of the surrounding vapor. As a result the 

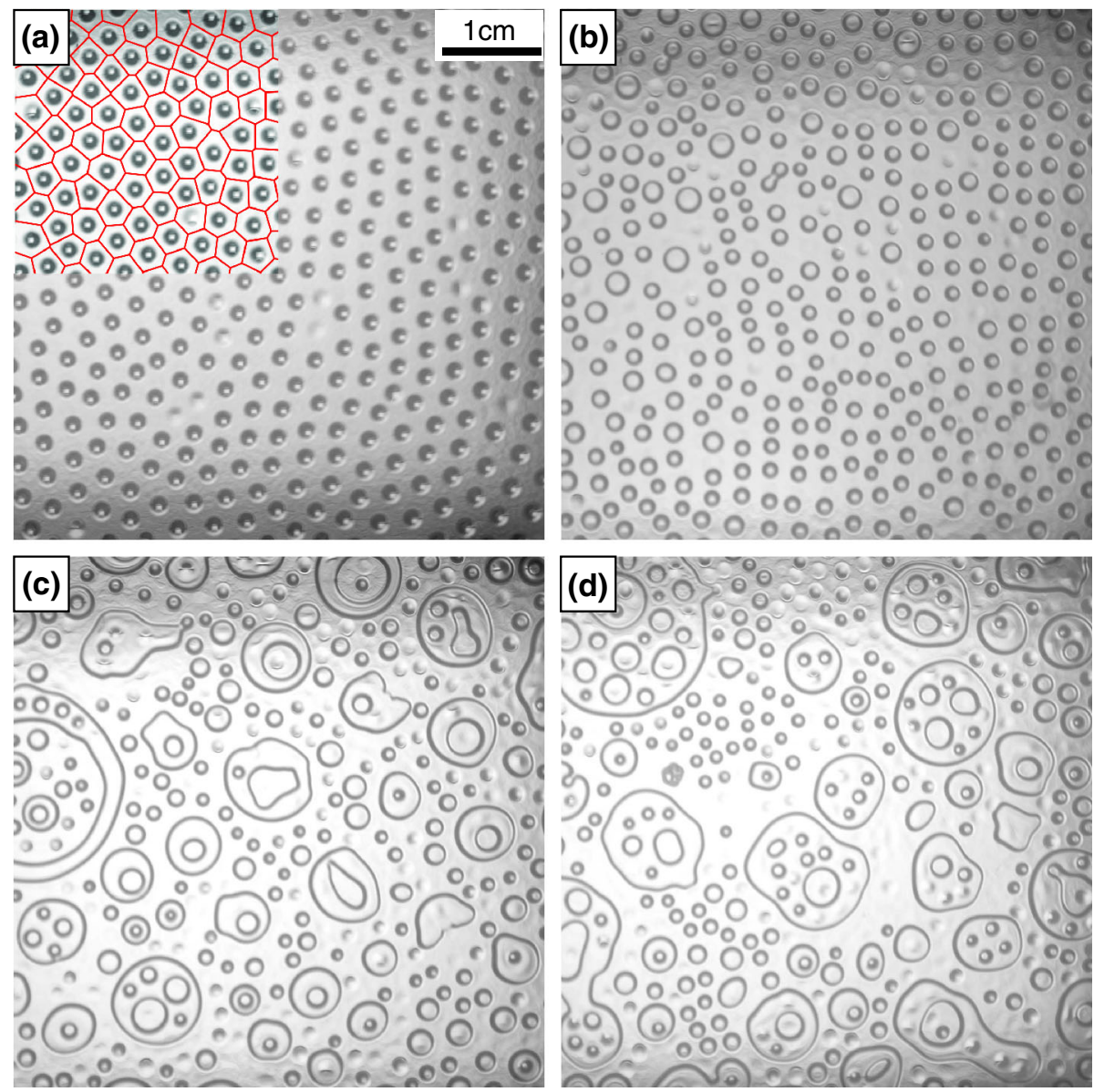

Fig. 3 Snapshots of Leidenfrost drops under different conditions. a $T_{t}=32.00^{\circ} \mathrm{C}, T_{b}=40.00{ }^{\circ} \mathrm{C}, P=28.4$ $\operatorname{bar}(\phi=0.88) . \mathbf{b} T_{t}=36.00^{\circ} \mathrm{C}, T_{b}=40.00^{\circ} \mathrm{C}, P=31.17 \operatorname{bar}(\phi=0.72) . \mathbf{c} T_{t}=36.00{ }^{\circ} \mathrm{C}, T_{b}=40.00^{\circ} \mathrm{C}$, $P=31.45$ bar $(\phi=0.62)$. d $T_{t}=36.00{ }^{\circ} \mathrm{C}, T_{b}=40.00{ }^{\circ} \mathrm{C}, P=31.72$ bar $(\phi=0.52)$. The red pattern overlayed on parts of the image in a mark the Voronoi lattice calculated for a quantitative analysis of the ordered pattern. Movies showing the drop dynamics for all four conditions can be found in the supplementary material

neighboring drops occasionally merged to form a larger drop which disturbed the arrangement of drops in its vicinity. The resulting large drop often shrunk again due to evaporation to a similar size as the two drops had before. New drops formed whenever larger areas without drop existed, for example when drops had merged previously.

Figure $3 b-d$ shows the effect of decreasing temperature fraction $\phi$ on the Leidenfrost drops. Please note, as $\phi$ was changed by increasing the pressure (i.e., a change in $T_{s}$ ), also the fluid properties changed slightly. However, the latter is minor and we attribute the main difference in observations to the change in $\phi$. As can be seen, the drop size on average increased as $\phi$ in the cell decreased, whereas the bigger drops in Fig. 3b formed through the merging of smaller drops. A further decrease in $\phi$ increases the lateral size of the drops, forming more laterally extended puddles of liquid hovering on a vapor cushion. These puddles formed chimney like structures inside them (Fig. 3c, d), as their sizes increased above the 
certain critical value $r_{c}$. We discuss the effect of $\phi$ on the sizes of the drops and puddles again in Sect. 3.5. We now discuss in more detail some of the phenomena described here.

\subsection{Chimney Formation}

Figure 4 shows the formation and disappearance of a chimney at $T_{t}=36.00^{\circ} \mathrm{C}, T_{b}=$ $40.00^{\circ} \mathrm{C}$ and $P=31.17$ bar $(\phi=0.72)$. The four Leidenfrost drops shown in the bottom half of Fig. 4a merged into a large drop. The resulting drop oscillated due to released surface energy that was transformed into kinetic energy (Fig. 4b). The measured oscillation time for this drop was $0.3 \mathrm{~s}$ which is of the same order of magnitude, but above the oscillation time for free oscillating drops $\tau \approx 0.1 \mathrm{~s}$ that can be computed as $\tau=\left(R^{3} \rho_{l} / \sigma\right)^{1 / 2}$, with $R$ being the size of the drop (the so called Rayleigh frequency) [20]. It seems plausible that the drop oscillation time here is larger than that of free drops as they are attached to the top plate, thus have less surface curvature at the same drop size and thus smaller Laplace pressure that forces the oscillation. Similar to free drops also in our case the oscillation time increases with the size of the drops. However, a quantitative analysis of the drop oscillation as a function of the size, density and surface tension is not the focus of this paper.

After the oscillation has stopped due to viscous friction, the resultant drop acquired a circular shape as shown in Fig. 4c. The drop was large enough to be flattened due to its own weight. We estimate a lateral diameter of $3.0 \mathrm{~mm}$, which can be compared to its height of less then $0.46 \mathrm{~mm}$ (i.e., the cell height). This size was above the critical size and as a consequence a chimney forms in its center which is clearly visible in Fig. $4 \mathrm{c}$ and $\mathrm{d}$. The diameter of the chimney increased over time as shown in Fig. 4c-e. The chimney reached its maximum size after a bit more than a second (Fig. 4e) before it started to decrease again as shown in Fig. 4ef. This cycle of expanding and shrinking of the chimney repeated over time (Fig. 4g-k) until the drop shrunk below the critical diameter for which chimney formation was not possible as shown in Fig. 4l. The drop had significantly shrunk over time due to evaporation as can be seen by comparing Fig. $4 \mathrm{c}$ and 1 . The evaporation continued until the drop had reached a stationary size, i.e., where evaporation and condensation rates were in balance.

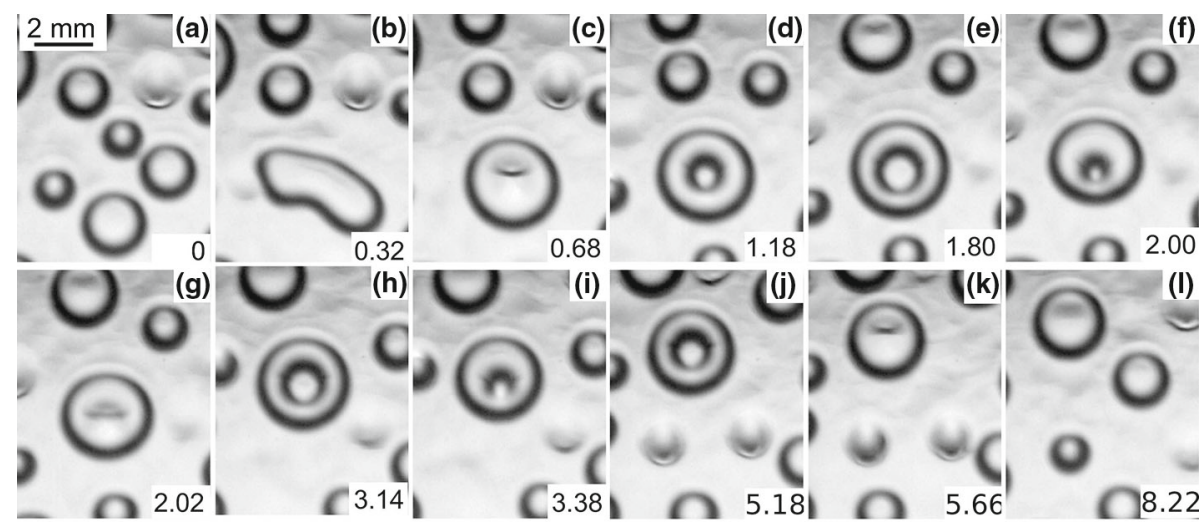

Fig. 4 Closeup of Leidenfrost drops at $T_{t}=36.00^{\circ} \mathrm{C}, T_{b}=40.00^{\circ} \mathrm{C}, P=31.17 \mathrm{bar}$ ( $\mathrm{same}$ conditions as in Fig. 3b). In the sequence the four drops at the lower part of the cell in a merge to one large drop (b and $\mathbf{c})$ that is above the critical size, so that bubbles occurs in them (d). The time in seconds is indicated in the bottom right corner of each image e-k chimney oscillations - expansion and shrinking of the chimney, $\mathbf{l}$ stable drop below the critical size with no chimney oscillation 
The oscillatory behaviour of the chimney was not reported in previous studies of Leidenfrost drops. Furthermore, this oscillatory behaviour was only observed in drops with only one chimney. A possible explanation for these oscillations can be found by considering the evaporation-condensation dynamics inside the bubble. At the beginning, the bubble increases in thickness due to evaporation and the resulting large pressure underneath the drop. This cools the upper part of the bubble which results in the condensation of $\mathrm{SF}_{6}$ vapor there. Consequently, the vapor pressure inside the bubble decreases, causing a reduction in the bubble size. Below a certain size the pressure in the bubble starts increasing again due to evaporation from the drop and the bubble expands again. This cycle repeats until the radius of the drop falls below the critical radius for bubble formation. In the experiment shown in Fig. $4\left(T_{t}=36.00^{\circ} \mathrm{C}, T_{b}=40.00^{\circ} \mathrm{C}, P=31.17\right.$ bar) we observed chimney oscillations with a period of $\tau_{c} \approx 1.4-1.6 \mathrm{~s}$. This time period is about five times larger than the transient capillary oscillations time period, $\tau=0.3 \mathrm{~s}$, after the merging of multiple drops (Fig. 4a and b). Similar chimney oscillations have also been found at a different mean temperature (and thus very different fluid parameters) as for example with $T_{t}=24.0^{\circ} \mathrm{C} ; T_{b}=30.0^{\circ} \mathrm{C}$; $P=23.72$ bar. Here, the drop oscillation time-period $\tau_{c}=1.3-1.6 \mathrm{~s}$, was very similar to the time-period for the experiments shown in Fig. 4. It appears that the time-period of the oscillation is independent of the material properties. Thus we conclude that capillary forces may not play a prominent role in the chimney oscillation. It is difficult however to estimate a time scale for chimney oscillations based on the proposed mechanism above due to the limitations in the measurement capability. In particular, the local heat transfer, evaporation, and condensation rates are required for estimating the time-scales of this mechanism. Raufaste and coworkers have measured the chimney formation and expansion for water drops in a thin Hele-Shaw cell [21]. There, it took just a few milliseconds for the chimney to grow to a few millimeter. While both our liquid and our system is different, we also see that the time for the chimney to grow is rather small compared to the typical oscillation time, suggesting that the slowest time scale in the oscillation process is related to the cooling and condensation rates that causes the chimney to collapse again.

Surprisingly, such oscillatory behaviour was not visible in drops with multiple chimneys. We can only speculate as to why this is the case, but certainly the larger amount of liquid that needs to be pushed to the sides when the bubble expands and pulled together when the bubble collapses must damp these oscillations due to its inertia.

\subsection{At Small Temperature Fraction $\phi$}

As more liquid was available, i.e., at smaller $\phi$, the drops and puddles increased in size. As the liquid puddles grew in size, the chimneys inside them either increased in size as well, or additional chimneys formed as a result. Figures 5, 6, and 7 show the dynamics of the drops for $\phi=0.62\left(T_{t}=36.00^{\circ} \mathrm{C}, T_{b}=40.00^{\circ} \mathrm{C}, P=31.45\right.$ bar, corresponding to Fig. $\left.3 \mathrm{c}\right)$. In Fig. 5a, we see a ring-like liquid puddle surrounding a central vapor area. Also this liquid puddle was not in contact with but levitated above the bottom plate. This observation is similar to confined Leidenfrost puddles in a Hele-Shaw cell with a cell gap significantly smaller than the capillary length $[22,23]$. There, however, the drop was confined in between two hot plates, thus a vapor cushion formed at the bottom and the top plate. Furthermore, the liquid rings observed in the Hele-Shaw experiments have a rather short lifetime. There, as the liquid ring (i.e., the puddle with a chimney) expands due to the excess pressure in the chimney, the size of the liquid rim becomes thinner and eventually becomes unstable due to Rayleigh-Plateau instability thus bursting into smaller droplets. The lifetime of the ring is further decreased due 

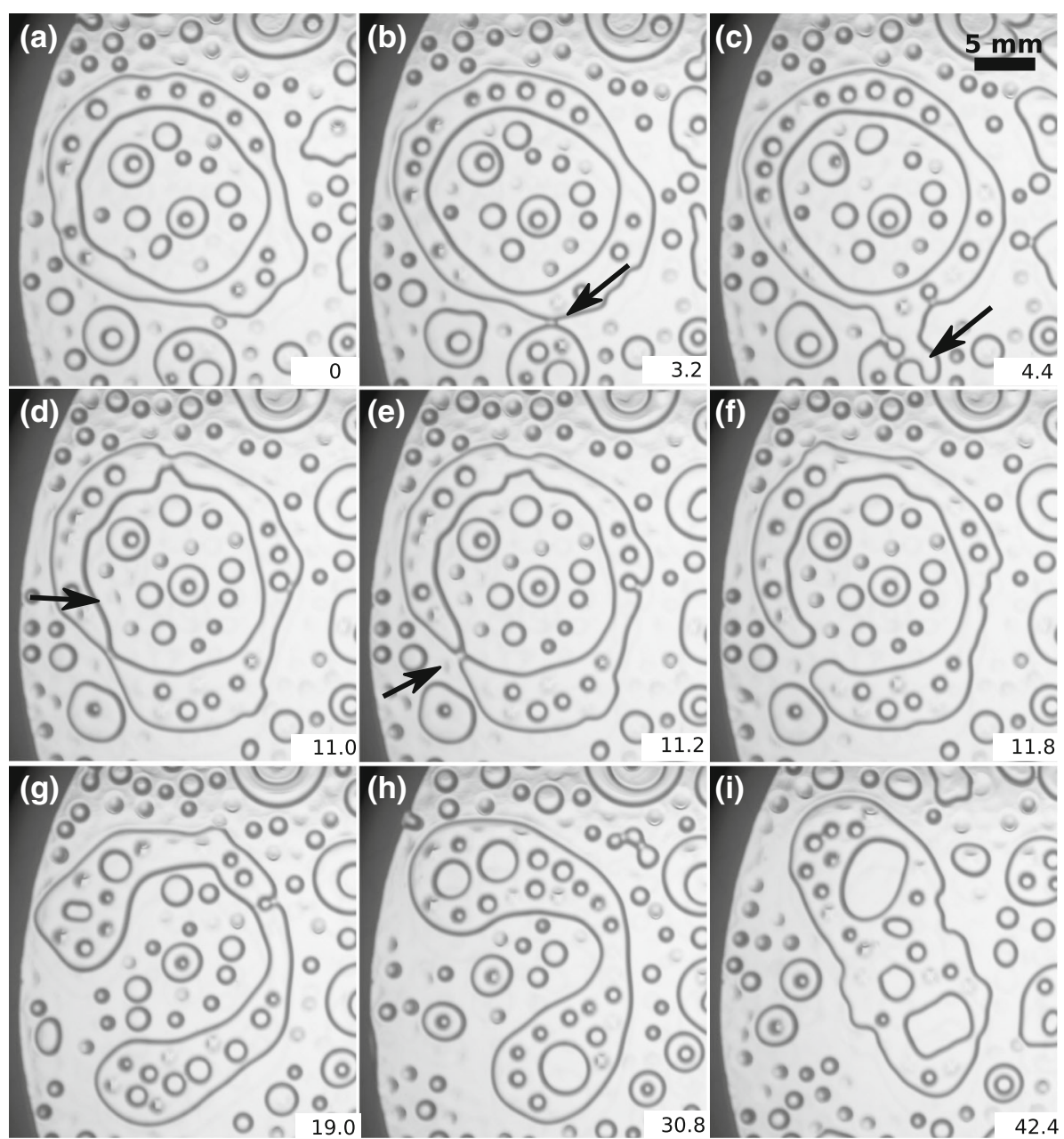

Fig. 5 Dynamics of a large ring-shape puddle in the Leidenfrost regime at $T_{t}=36.00^{\circ} \mathrm{C}, T_{b}=40.00^{\circ} \mathrm{C}$, $P=31.45$ bar $(\phi=0.62)$. Ring-shaped puddle with multiple chimneys $(\mathbf{a})$. The black arrows mark the merging of the puddle with a neighboring smaller puddle (b), the release of a bubble (c), the appearance of a new drop (d), and the pinch-off of the ring puddle (e). Rearrangement of the ring-shaped puddle after pinch-off (f-i). The time stamp (in seconds) is indicated in the bottom right corner of the image. See also the corresponding movie in the supplementary material

to slow but continuous evaporation of liquid. In contrast, the liquid rings in our experiments are connected to the cold top plate and thus condensation and evaporation are in balance. Any excess build-up of pressure is prevented by the condensation of the vapor. Therefore, the radial pressure gradient across the liquid ring is quite small after the formation of the chimney. This prevents the radial expansion of the liquid ring. Furthermore, merging of an expanding puddle with neighboring drops and puddles adds liquid and prevents the ring from becoming too thin. The large gas area inside the ring originates from a chimney that formed in the middle of an unstable puddle, and that has since been grown together with the puddle. Small holes (chimneys) have formed inside the liquid ring. Within the central vapor area, multiple drops formed and some of them had sizes above the critical size such that new chimneys formed inside them. 

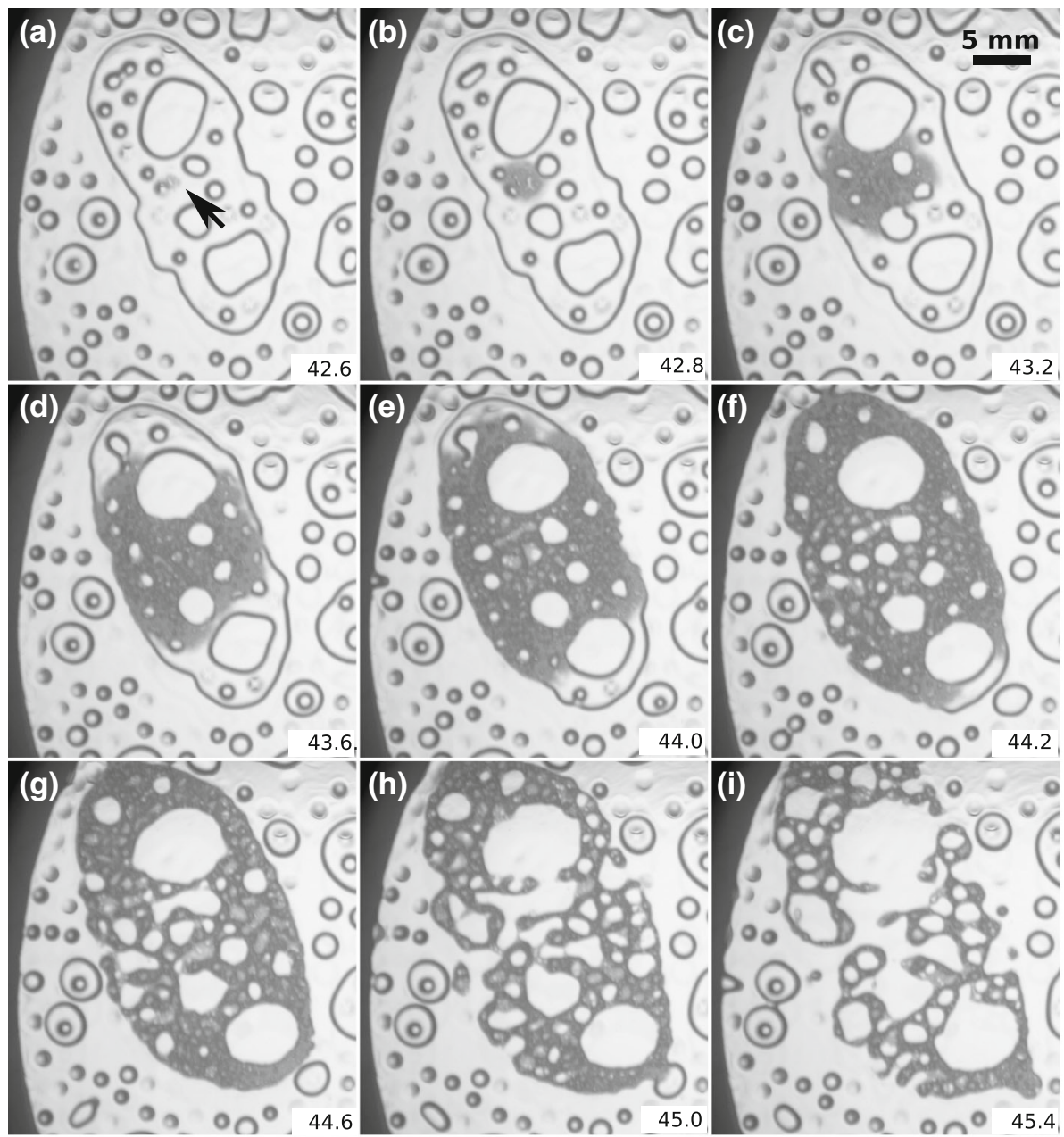

Fig. 6 Boiling of a drop. (1) Location where boiling started. The time stamp (in seconds) is indicated in the bottom right corner of the image. The time stamp is time duration in seconds from Fig. 5a. Experimental conditions were as in Fig. $5\left(T_{t}=36.00{ }^{\circ} \mathrm{C}, T_{b}=40.00^{\circ} \mathrm{C}, P=31.45 \mathrm{bar}, \phi=0.62\right)$. See also the corresponding movie in the supplementary material

The puddles in Fig. 5 were not steady but deformed over time, merged with neighboring drops and puddles, while at the same time new chimneys and drops appeared while others disappeared due to condensation and evaporation. As an example, we see in Fig. 5b-d the merging of the ring shaped puddle with another puddle close by. First contact occurred in Fig. 5b, with the formation of a liquid bridge between both liquid puddles. As a result of the merger, the large ring-like puddle was re-arranging its overall shape, whereas some of the chimneys (bottom center of the image) were exiting the liquid puddle (Fig. 5c). We also observed the formation of new drops from the liquid layer at the top plate inside the central area that was surrounded by the ring-like puddle (Fig. 5b, c). The sequence in Fig. $5 d-i$ shows the exiting of the big chimney from the ring-like drop. The ring first became very thin as seen in the lower left quarter of Fig. 5d, before it finally broke (Fig. 5e). Surface tension immediately retracted the open ends of the liquid in order to minimize the surface 

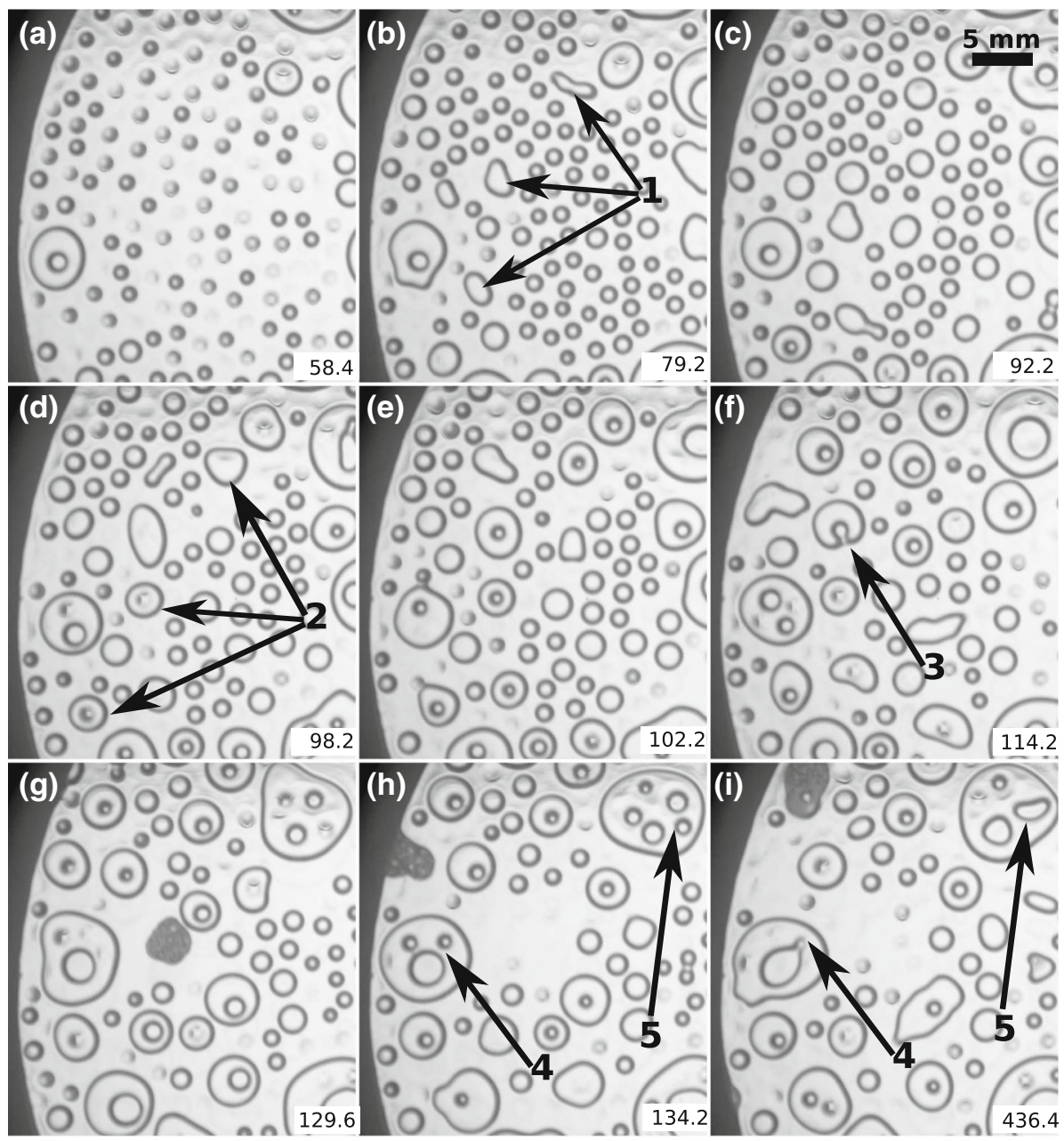

Fig. 7 Formation of large drops with chimney. See text for details. (1) Merged drops exhibiting capillary oscillations. (2) Formation of chimney in a few sufficiently large drops. (3) Exit of chimney from a drop. (4) Merging of small chimney with a large chimney. (5)Merging of two chimneys of similar dimensions. The time stamp represents the number of seconds from Fig 5a. Experimental conditions were as in Figs. 5 and $6\left(T_{t}=36.00^{\circ} \mathrm{C}, T_{b}=40.00^{\circ} \mathrm{C}, P=31.45 \mathrm{bar}, \phi=0.62\right)$. See also the corresponding movie in the supplementary material

area (Fig. 5f), and rearranged the half-ring into a more compact shape (Fig. 5g-i). In this process, the drops within the big chimney were pushed away from their initial positions and outside of the areas previously encircled by the liquid ring. Some of these drops merged with the large ring like puddle. The number of chimneys in the ring puddle decreased post the exit of the big chimney due to merging of some of the chimneys. As a consequence the diameter of the chimneys had increased (Fig. 5h, i). The initial instability causing the liquid ring to break is similar to a Plateau-Rayleigh instability, where deviations of the stable liquid surface can be amplified when the wavelength of the perturbation is sufficiently large. However, here we do not have a free liquid tube as the liquid is attached to the top plate. Furthermore, pressure variations due to the vapor flow may play an important role in destabilizing the initial liquid structure. 
Figure 6 is a continuation of the sequence presented in Fig. 5 showing the boiling of a liquid puddle. Boiling occurred first at a small well defined location (marked in in Fig. 6a), where the heat transport must have been increased significantly. As a result, the puddle started to boil after it came into contact with the bottom plate. Over time the entire liquid puddle started to boil. The spreading of the boiling region is shown in the image sequence in Fig. 6a-f. The structure of the relatively large chimneys were preserved until the boiling of the puddle was completed. Note that boiling only occurred in a single connected puddle, while surrounding drops and puddles were not affected. While it is not quite clear why the liquid touched the bottom plate as it was floating on its vapor for several seconds, it seems plausible that the large size of the liquid puddle caused a local decrease of the surface temperature of the bottom plate as there, the cooling is large and the replenish of heat by diffusion is insufficient to hold the temperature above $T_{L}$ If this happens the vapor pressure beneath the puddle is not sufficient for sustaining the weight of it. As a consequence the liquid touches the bottom plate and starts boiling immediately due to the enhanced heat transport. The boiling event locally increased the vapor pressure significantly and this caused the neighboring drops to move away from the boiling drop. We are aware that this is a speculation on our side and needs to be substantiated by further careful experiments and theoretical calculations.

Figure 7 is a continuation of the sequence presented in Figs. 5 and 6. The boiling of a large mass of liquid resulted in a very high supersaturation locally. The vapor condenses on the top plate and undergoes immediately a Rayleigh-Taylor like instability as shown in Fig. 7a. The drops formed grew over time through vapor condensation and also by merging with other drops. Some of the merged drops are shown in Fig. 7b. They exhibited capillary oscillations after the merger event similar to the one reported before in Sect. 3.3 (see Fig. 4). Over time the diameter of these drops increased further by merging with other drops in their neighborhood (Fig. 7c, d). Above a critical diameter the drops started to form a chimney as shown in Fig. 7d. As the diameter increased further, multiple chimneys appeared in the drop (or puddle). One such puddle is shown in Fig. 7d. As the drops and puddles expanded, the chimneys also migrated towards the edge of the drop and eventually exited from the drop. This was discussed already in the context of Fig. 5. These chimneys also grew by merging with other chimneys in their neighborhood. Figure $7 \mathrm{~h}$, i depict the merging of a large chimney with a smaller chimney. The drops of various sizes merged together over time and resulted in puddles with several chimneys, like the one shown in Figs. 5 and 6. The process of formation of chimneys and exiting from the drop occurred repeatedly until the puddle touches again the bottom plate and boils away. As was shown in Fig. 3, the size of these puddles increased with the vapor pressure in the cell.

\subsection{Critical Drop Size and the Chimney Instability}

For a quantitative analysis, we measured the sizes of drops without a chimney and of the drops and puddles with exactly one chimney. For drops without a chimney a circle was fitted to the minimal grey-values in the image (i.e., the dark ring) surrounding a drop, while for larger puddles another computer algorithm was used that finds the exact shape of the boundary of the puddle. The area that is enclosed by the boundary $A$, is then measured and an effective puddle radius is calculated as $r_{p}=\sqrt{A / \pi}$. In this procedure, only drops with a clear dark border were considered, i.e., only drops reaching to the bottom plate. For the statistical analysis discussed below we measured at least 200 drops of each kind (i.e., with and without a chimney) as well as for each experimental condition. 


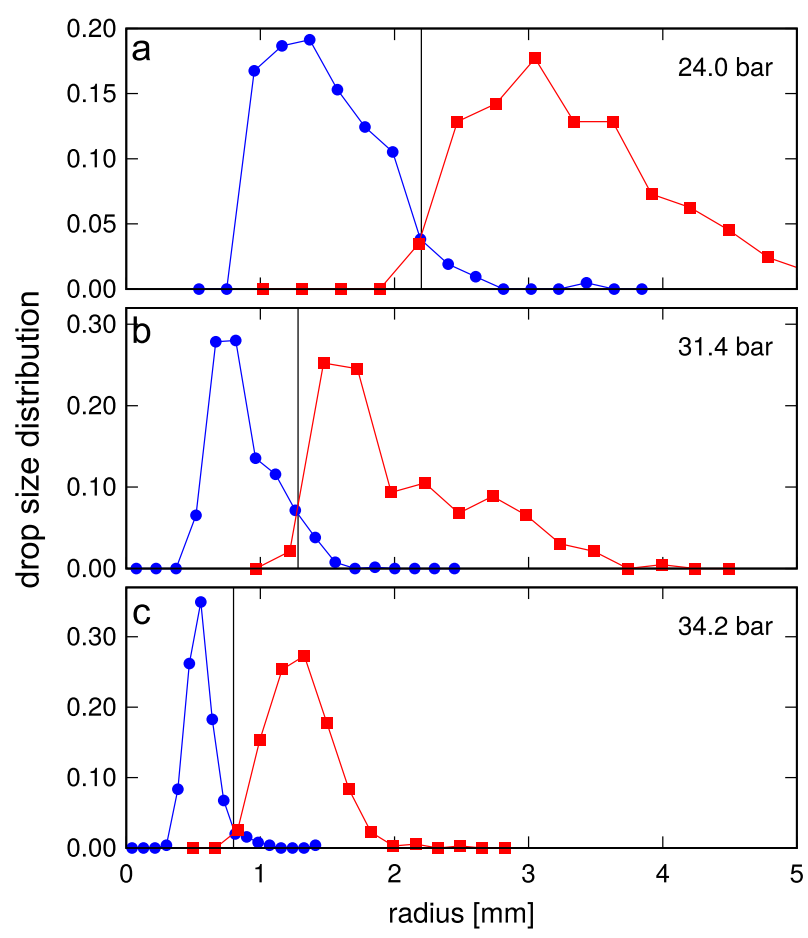

Fig. 8 Size distribution of the drops without a chimney (blue bullets) and drops with exactly one chimney (red squares) for three different pressures. The solid black vertical lines mark the location where both size distributions overlap, which is considered to be the critical drop size for the formation of a single chimney (Color figure online)

The normalised size distributions of the drops under different pressures are shown in Fig. 8. The blue bullets mark the sizes of the drops without, while the red bullets mark the sizes of the drops with exactly one chimney. One clearly sees that there is very little overlap in these distributions, suggesting that there is a critical size of drops (or liquid puddles), above which they are unstable and form chimneys. In order to determine the critical size, we connect neighboring data points with lines and mark as critical size where the distributions for the drops without and with a single chimney cross (marked with black vertical lines in Fig. 8). Due to the rather low sample size the statistics reported here is subject to significant levels of uncertainty. Nevertheless, we plot in Fig. 9a the critical drop size as a function of the capillary length $\lambda$, with error bars reflecting their uncertainty. The capillary length was calculated based on the saturation temperature at the pressure in the cell (see Table 1). We see that within the error bars, the critical drop size is proportional to the capillary length, as it has been found for Leidenfrost drops [5]. For the critical drop radius we found $r_{c} \approx 5.1 \lambda$ which is about $25 \%$ larger than the critical size of freely levitating Leidenfrost puddles $R_{c} \approx 4 \lambda[2,5]$. In contrast to a freely levitating drop, in our case the region between the outer gas pocket and the outer drop region has an opposite curvature, and in addition the drop is attached to the top plate (see Fig. 2b). This may result in a higher critical radius of hanging Leidenfrost drops.

The color of the data points shown in Fig. 9a represent the temperature fraction $\phi$ at which the data were acquired. We see that while the influence of $\phi$ is rather weak, it appears 

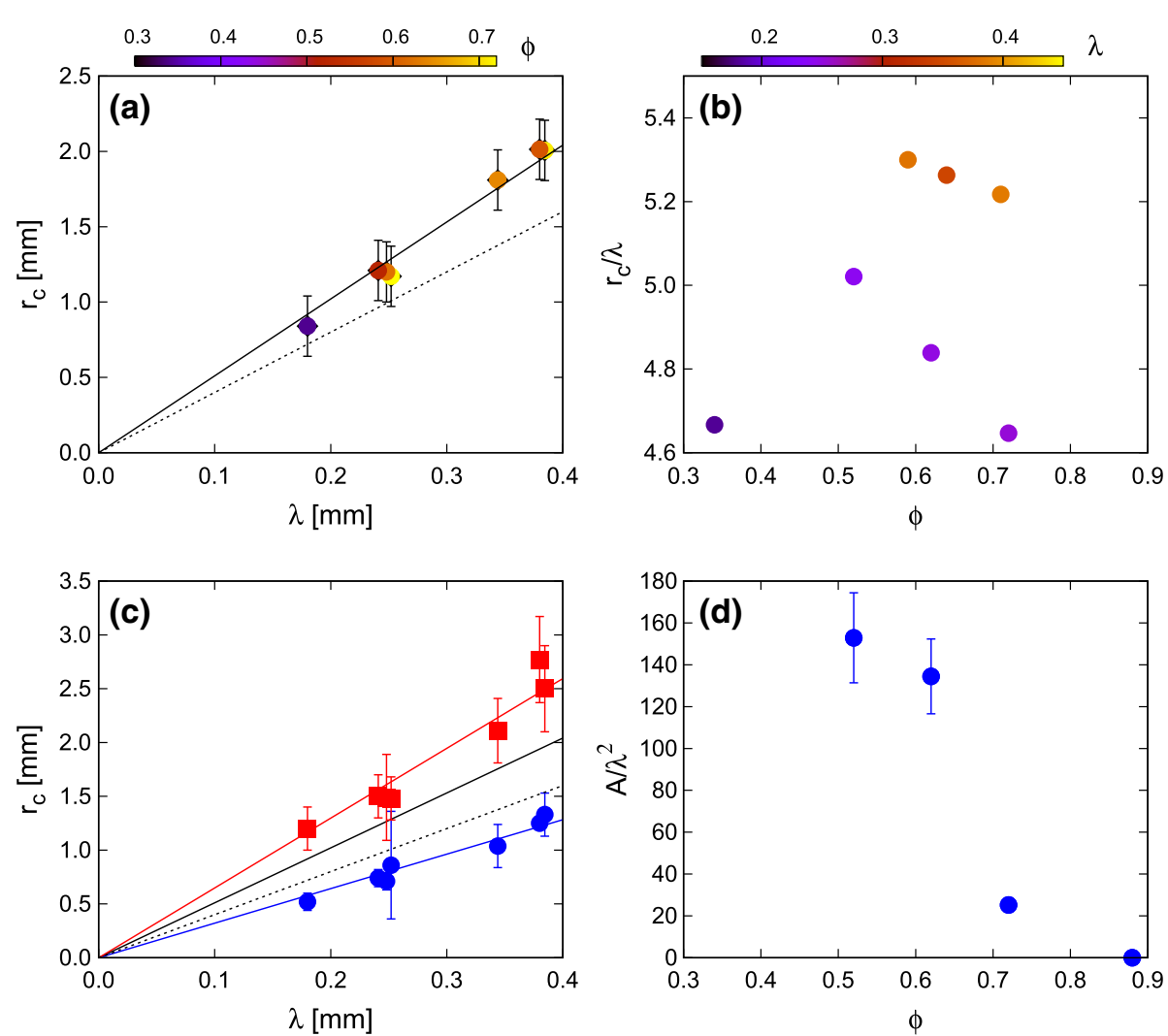

Fig. 9 Statistical quantities of drop and puddle sizes. a Critical drop size $r_{c}$ for the formation of a single chimney as determined from the particle size distributions as a function of the capillary length $\lambda$. The color code of each point reflects the temperature fraction $\phi$. The solid black line is a linear fit to the data resulting in $r_{c}=5.1 \lambda$. The dotted line marks the critical drop size for freely levitating Leidenfrost drops [2,5]. b The same data, but normalised by $\lambda$ and plotted against the temperature fraction $\phi$. Color of the points reflect $\lambda$. The uncertainty is larger than the variation of the data points. We thus omitted error bars for better visibility. c Maximum of the particle size distribution of drops without (blue) and with exactly one chimney (red) as a function of $\lambda$. The black solid and dashed lines are the same as in a. $\mathbf{d}$ Dimensionless average area of puddles and drops as a function of $\phi$ (Color figure online)

that points with a larger $\phi$ show slightly smaller $r_{c}$. We further investigate this finding in Fig. $9 \mathrm{~b}$, where we plot the ratio $r_{c} / \lambda$ against $\phi$. In this plot we colored the data points as a function of $\lambda$. We see that for a given $\lambda$ (e.g., only the orange points) an increase in $\phi$ leads to a small decrease in $r_{c} / \lambda$. As this decrease is within the margin of error, and there is no obvious physical explanation for this, we believe that this may be due to a measurement bias. When $\phi$ is small, most drops and puddles are small and only a few drops are observed with a chimney (as shown in Fig. 3). Therefore, the puddles that are considered are in general small and barely above $r_{c}$. While this would not be a problem with a sufficient amount of data, since we only consider about 200 drops for the statistics, the drop size distribution is not very smooth and the resolution (i.e., the bin size) is rather coarse here. Furthermore from the color code in Fig. 9b, we can see that although here $r_{c}$ was normalised by $\lambda$, there is still a clear $\lambda$ dependency in the data suggesting a small higher-order (quadratic) dependency of $r_{c}$ on $\lambda$. 

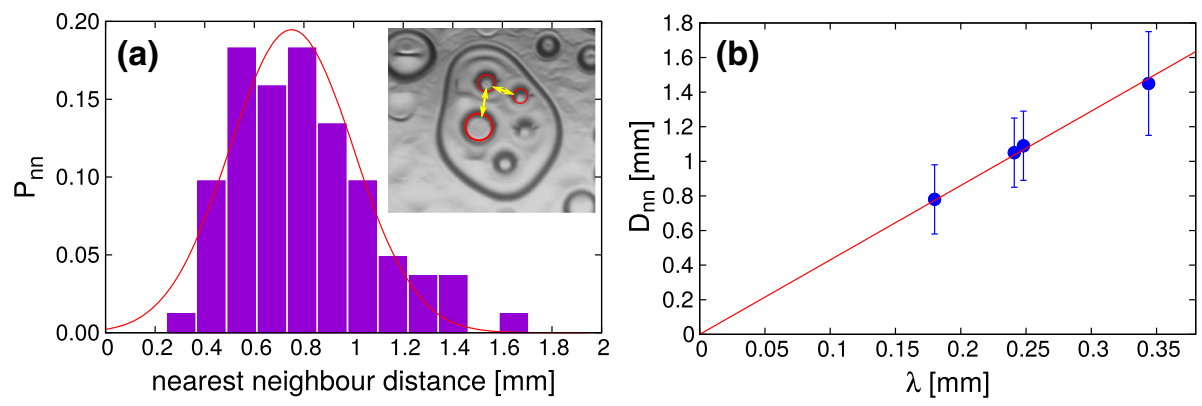

Fig. 10 Nearest distance between neighboring chimneys. $\lambda$. a Normalised distribution of the nearest distances $P_{n n}$ for $T_{t}=40^{\circ} \mathrm{C}, T_{b}=42{ }^{\circ} \mathrm{C}, \mathrm{P}=34.2$ bar $(\phi=0.34)$. The red curve represents a normal distribution for comparison. b Averaged nearest distances $D_{n n}$ as a function of the capillary length. Blue bullets mark measurements, while the red line is a linear fit with slope 4.3 to the data. Inset in a shown an example image. The nearest distance was measured from the rim of a chimney to the rim of its nearest neighbor, i.e., the yellow arrows (Color figure online)

Figure 9c shows also the maxima of the drop size distribution for drops without (blue) and drops with exactly one chimney as a function of $\lambda$. Also these quantities are in first order proportional to $\lambda$ with coefficients of 3.21 and 6.48 respectively, such that $r_{c}$ is for a given $\lambda$ roughly in the middle between the drop sizes where the two size distribution are maximal. This should not come as a surprise as $r_{c}$ was assumed to be right where the size distribution functions cross. We note that there are barely drops visible of radial sizes close to $r_{c}$. This is since drops without a chimney become unstable when they approach $r_{c}$ and the occurring chimney increases the drop size significant as fluid is pushed to the side.

While we see already from Fig. 3 that a decrease in the temperature fraction $\phi$ causes on an average larger drops and puddles to occur, we wanted to quantify this effect and plot in Fig. 9d the average area occupied by a single puddle $(A)$ normalised by $\lambda^{2}$ as a function of $\phi$ for the four cases shown in Fig. 3. The puddles area was calculated based on the largest extent of a connected liquid volume. A thin liquid ring surrounding a large gas area as in Fig. 5a would count as a single large puddle. From the plot the trend to larger drops with decreasing $\phi$ is apparent. Note that for the smallest temperature fraction $\phi=0.52$ the average area is not much larger than for the next data point at $\phi=0.62$. In fact we would have expected a significantly larger value as puddles are still much smaller than the cell diameter and there is no apparent reason why the slope $-\partial\left(A / \lambda^{2}\right) / \partial \phi$ should decrease. However, we observe that larger puddles are destroyed by boiling events as shown in Fig. 6. After such a boiling event many small drops form where a larger puddle was destroyed through boiling. While boiling events are rare for the point at $\phi=0.62$ they do occur more frequently for the point at $\phi=0.52$. While these events significantly lower the average puddle size, they are not a well defined function of $\phi$. As was pointed out earlier, the boiling event was merely a sign of a finite thermal diffusivity of the bottom plate in the experiment. We note that other datasets listed in Table 1 show boiling at even smaller puddle sizes and thus are excluded in Fig. 9d.

One can learn something about the instability that leads to the formation of chimneys by quantifying the distances between neighboring chimneys. This is done in Fig. 10. Chimneys occur and grow in sizes due to evaporation but also by merging with other neighboring chimneys. As a result, different chimneys have different sizes. Therefore, distances between the centers of the chimneys do not contain information about the instability leading to chimney formation or the stability of their spatial arrangement. We therefore, consider the width of the liquid between the boundaries of nearest chimneys, as marked with yellow arrows in Fig. 10. 
To find the nearest neighbour, we determine the location and sizes of all chimneys inside a multi-chimney puddle. We then identify for every chimney its nearest neighbor and use this distance for the statistical analysis. Hereby we ensure not to double count distances. For this analysis more than 200 chimneys were considered. Figure 10a shows the distribution of the nearest neighbor distances $P_{n n}$ for $T_{t}=40^{\circ} \mathrm{C}, T_{b}=42^{\circ} \mathrm{C}, \mathrm{P}=34.2 \mathrm{bar}(\phi=0.34)$. For comparison we also show a normal distribution (red line). One sees that $P_{n n}$ is a bit skewed to the left, compared to the normal distribution. It seems that very small liquid rims become unstable due to capillary forces, causing neighboring chimneys to merge and therefore are not being observed. If the distance becomes too large, a chimney instability occurs that results in the formation of a new chimney. This process however is much slower than the merging of chimneys which is driven by capillary forces as can be seen from Fig. 4 and discussed in Sect. 3.4. As a result larger distances can be observed even though they might undergo a chimney-instability later on. The averaged nearest distance $D_{n n}$ reflects an optimal distance that is right in between these two instabilities.

In Fig. 10b we show $D_{n n}$ as a function of the capillary length $\lambda$. A fit to the data reveals a proportionality between the nearest neighbor distance $D_{n n}$ and the capillary length of the form $D_{n n}=4.3 \lambda$. This can be compared with measurements reported by Maquet et al. [24], who found a relation $D_{c h}=7.89 \lambda$. However, they defined $D_{c h}$ as the distance of nearest chimneys measured between the centers of each chimney. For small chimneys that have just emerged, the chimney diameter is comparable to the width of the liquid rim between two adjacent chimneys as can be seen in Fig. 10a, thus $D_{c h} \approx 2 D_{n n}$. Therefore, the finding by Maquet et al. is in accordance with our observations provided the difference in definitions is taken into consideration. We also want to point out that our coefficient is surprisingly close to the coefficient between capillary length and the critical drop radius for the formation of chimneys in freely levitating Leidenfrost puddles as discussed in [2,5]. While we believe that this is rather a mere coincidence, we note that this is consistent with the fact that the nearest neighbor distances increases smaller with $\lambda$ than the critical size for the chimney instability. The nearest neighbor distance has firstly only an upper bound with the chimney-instability, while the lower bound is some sort of Rayleigh-Plateau instability.

\section{Summary}

In this paper, we report on observations in pressurized sulfur-hexafluoride trapped between two horizontal plates, with the lower one warmer and the upper one colder than the liquidvapor transition temperature. We investigated the shape of drops and condensation pattern as a function of the temperature ratio $\phi$, the pressure, and the temperature difference between both plates. For not too large $\phi$ the liquid drops and puddles do not boil but levitate on top of a thin vapor cushion, similar to Leidenfrost drops, while still being connected to a thin liquid layer underneath the top plate. As the drops grow above a critical size, vapor bubbles (chimneys) form inside them. Similar chimneys have been observed already in freely levitating, classical, Leidenfrost drops [2,5]. There, the formation of chimneys is attributed to an instability of the vapor cavity below the drop, similar to a Rayleigh-Taylor instability in puddles significantly larger than the capillary length. This instability causes the formation of a dome like structure in the center with a layer of liquid on the top. Over time this dome bursts resulting in the formation of a liquid torus that however closes again [2,5]. In our experiments as the drops and puddles were still connected with the top plate, we did not observe any such ruptures 
(Figs. 4-7). Instead, as reported above (Fig. 4), the chimneys can oscillate, grow, shrink, or exit the drop at its edges (see e.g., Figs. 5-7).

For larger drops and puddles we observe multiple chimneys, or chimneys that grow large enough to enable the creation of new drops inside them. If puddles become to large, cooling can decrease the bottom plate temperature locally below the Leidenfrost temperature, such that liquid gets into contact with the plate, resulting in an enhanced heat flux and consequentially vigorous boiling of the puddle.

Note that the reported phenomena here are influenced by the convection in the gas due to buoyancy. Despite the rather small height of the gas layer, the estimated Rayleigh numbers are of the order of $\mathrm{Ra} \approx 10^{5}$ and thus the gas flow is expected to be turbulent. This can also be observed in the corresponding movies in the supplementary material as a jitter in the gas phase. While there is no flow visible in the liquid phase, we also expect flow there. In particular, the liquid is driven by Marangoni forces caused by temperature differences across the liquid-gas interface. In our experiments, the Marangoni number, which compares thermocapillary and viscous forces is of the order of $\mathrm{Ma} \approx 10^{5}$. While this shows that certainly convection is possible inside the liquid drops and puddles, it is unclear how much Marangoni forces influence the observed phenomena. In fact, the gas flow in the thin layer underneath each drop also drives convection inside the liquid via shear forces. Therefore one needs to compare the Marangoni forces with these shear forces in order to estimate their influence. For that one would need the knowledge about the flow underneath the liquid.

The Leidenfrost phenomena reported in this paper are qualitatively similar to observations with liquids like water in air. Using $\mathrm{SF}_{6}$ under regulated pressure has the advantage that we can easily change relevant material parameter, such as the surface tension or the density ratio, by changing the pressure. In example, in this way it is rather easy to attain several chimneys in a single drop when the pressure is high and the surface tension is small.

In summary our experiments give a first glimpse of the pattern formation phenomena associated with Leidendrost effects in a shallow Rayleigh-Bénard cell. Further detailed studies are needed to gather a quantitative understanding of these very interesting phenomena.

Acknowledgements Open access funding provided by Max Planck Society. We acknowledge support from the Max Planck University Twente Center for Complex Fluid Dynamics.

Open Access This article is distributed under the terms of the Creative Commons Attribution 4.0 International License (http://creativecommons.org/licenses/by/4.0/), which permits unrestricted use, distribution, and reproduction in any medium, provided you give appropriate credit to the original author(s) and the source, provide a link to the Creative Commons license, and indicate if changes were made.

\section{References}

1. Leidenfrost, J.G.: On the fixation of water in diverse fire. Int. J. Heat Mass Transf. 9(11), 1153 (1966)

2. Quéré, D.: Leidenfrost dynamics. Ann. Rev. Fluid Mech. 45(1), 197 (2013)

3. Tong, L., Tang, Y.S.: Boiling and Heat Transfer in Two-Phase Flow, Boiling and Heat Transfer in TwoPhase Flow. Taylor and Francis, Bristol, PA (1997)

4. Nikolayev, V.S., Beysens, D.A.: Boiling crisis and non-equilibrium drying transition. EPL (Europhys. Lett.) 47(3), 345 (1999)

5. Biance, A.L., Clanet, C., Quéré, D.: Leidenfrost drops. Phys. Fluids 15(6), 1632 (2003)

6. Emmerson, G., Snoek, C.: The effect of pressure on the Leidenfrost point of discrete drops of water and freon on a brass surface. Int. J. Heat Mass Transf. 21(8), 1081 (1978)

7. Bernardin, J.D., Mudawar, I.: Film boiling heat transfer of droplet streams and sprays. Int. J. Heat Mass Transf. 40(11), 2579 (1997) 
8. Chaves, H., Kubitzek, A.M., Obermeier, F.: Dynamic processes occurring during the spreading of thin liquid films produced by drop impact on hot walls. Int. J. Heat Fluid Flow 20(5), 470 (1999)

9. Tran, T., Staat, H.J.J., Prosperetti, A., Sun, C., Lohse, D.: Drop impact on superheated surfaces. Phys. Rev. Lett. 108, 036101 (2012)

10. Snoeijer, J.H., Brunet, P., Eggers, J.: Maximum size of drops levitated by an air cushion. Phys. Rev. E 79, 036307 (2009)

11. Sobac, B., Rednikov, A., Dorbolo, S., Colinet, P.: Leidenfrost effect: accurate drop shape modeling and refined scaling laws. Phys. Rev. E 90, 053011 (2014)

12. de Bruyn, J.R., Bodenschatz, E., Morris, S.W., Trainoff, S., Hu, Y., Cannell, D.S., Ahlers, G.: Apparatus for the study of Rayleigh-Bénard convection in gases under pressure. Rev. Sci. Instrum. 67, 2043 (1996)

13. Plapp, B.B., Egolf, D.A., Bodenschatz, E., Pesch, W.: Dynamics and selection of giant spirals in RayleighBénard convection. Phys. Rev. Lett. 81(24), 5334 (1998)

14. Weiss, S., Seiden, G., Bodenschatz, E.: Resonance patterns in spatially forced Rayleigh-Bénard convection. J. Fluid Mech. 756, 293 (2014)

15. Lemmon, E.W., Huber, M.L., McLinden, M.O.: NIST standard reference database 23: reference fluid thermodynamic and transport properties-REFPROP, Version 9.1. National Institute of Standards and Technology, Standard Reference Data Program, Gaithersburg (2013)

16. Chandrasekhar, S.: Hydrodynamic and Hydromagnetic Stability. Dover, New York (1981)

17. Fermigier, M., Limat, L., Wesfreid, J., Boudinet, P., Quilliet, C.: Two-dimensional patterns in RayleighTaylor instability of a thin layer. J. Fluid Mech. 236, 349 (1992)

18. Tsimring, L.S.: Penta-Hepta defect motion in hexagonal patterns. Phys. Rev. Lett. 74, 4201 (1995). https://doi.org/10.1103/PhysRevLett.74.4201

19. Bestehorn, M., Merkt, D.: Regular surface patterns on Rayleigh-Taylor unstable evaporating films heated from below. Phys. Rev. Lett. 97, 127802 (2006)

20. Andrieu, C., Beysens, D.A., Nikolayev, V.S., Pomeau, Y.: Coalescence of sessile drops. J. Fluid Mech. 453, 427438 (2002)

21. Raufaste, C., Celestini, F., Barzyk, A., Frisch, T.: Hole growth dynamics in a two dimensional Leidenfrost droplet. Phys. Fluids 27(3), 031704 (2015). https://doi.org/10.1063/1.4916622

22. Celestini, F., Frisch, T., Cohen, A., Raufaste, C., Duchemin, L., Pomeau, Y.: Two dimensional Leidenfrost droplets in a Hele-Shaw cell. Phys. Fluids 26(3), 032103 (2014)

23. Raux, P.S., Dupeux, G., Clanet, C., Qur, D.: Successive instabilities of confined Leidenfrost puddles. EPL 112(2), 26002 (2015)

24. Maquet, L., Brandenbourger, M., Sobac, B., Biance, A.L., Colinet, P., Dorbolo, S.: Leidenfrost drops: effect of gravity. Europhys. Lett. 110, 24001 (2015)

Publisher's Note Springer Nature remains neutral with regard to jurisdictional claims in published maps and institutional affiliations. 